\title{
AN EVALUATION FRAMEWORK FOR POWER ELECTRONIC DEVICE MODELS USING DECISION MAKING UNDER UNCERTAINTIES
}

\author{
C. J. KIM ${ }^{1}$ and PETER BOFAH ${ }^{2}$ \\ ${ }^{1}$ Department of Electrical Engineering, Howard University, Washington DC 20059, USA, ckim@ @oward.edu \\ ${ }^{2}$ Department of Electrical Engineering, Howard University, Washington DC 20059, USA, bofah@cldc.howard.edu
}

\begin{abstract}
The uncertainty in modeling and simulation in power device comes from many different sources. This uncertainty brings a question of how to choose the best-fit model for a specific purpose and environment of a circuit designer. This paper proposes a numerical framework of decision making under incomplete knowledge, which quantifies the uncertainty of models and allows circuit designers to know the realistic expectations of the performance and, thus provides the level of appropriateness of the models. Case studies are performed for practical applications of the framework.
\end{abstract}

Key Words: Power Device Modeling, Model Evaluation, Uncertainty Reasoning

\section{INTRODUCTION}

Power electronics is one of the broadest growth area in electrical technology and its many and new applications are made possible by improvements in semiconductor devices and by better understanding of the circuits. Presently, power electronic circuit design practice relies heavily on extensive testing of components, subsystems and devices. With rapidly increasing computational capability, the modeling and simulation-based design is taking on increased responsibility for the success of new engineering systems. However, all models are only abstractions of the real systems and their performances are greatly dependent on the assumptions. As different models with different assumptions were introduced, the circuit designers face evaluation problems. The complex behavior of electronic devices prohibits circuit designers from evaluating the proper inclusion of model physics and from determining the best-fit model to fulfill their circuit simulations. Therefore, for circuit designers, there are practical needs; the fitness and uncertainties of models must be assessed before simulation process. This paper addresses a practical issue relating to the quantitative evaluation of the uncertainty.

This paper proposes a numerical framework of decision making under incomplete knowledge, which quantifies the uncertainty of models and allows circuit designers to know the realistic expectations of the performance and, thus provides the level of appropriateness of the models. In other words, circuit designers are able to select a model that is most appropriate to the intended circuit simulation purposes. The framework quantifies circuit designer's favorite level on the performance criteria, and calculates the range of the model-fit index of the candidate models.

This paper is organized as follows. We present the uncertainty problems surrounding the power device models in the next section. Then, we discuss the framework of decision-making under incomplete knowledge to manage the uncertainty. In section 4, we present two case studies employing the framework for practical applications. The discussions and conclusions follow.

\section{UNCERTAINTY IN POWER DEVICE MODELS}

The uncertainty in modeling and simulation in power device comes from many different sources such as idealization, approximation, simplification, linearization, truncation, and others of the device physics and numerical computations. As different models with different assumptions were introduced for power semiconductor devices, the circuit designers who just want to use most appropriate model for their simulation purposes, face problems of selecting the best-fit model, among many different models proposed, for a specific purpose and objective. Practically, there is a question: which of these models to adopt for their computer-aided design and analysis to simulate their circuits?

Among the issues in power device models, the performance criteria are the most important ones regarding the problems of model evaluation and adoption. The most frequently mentioned criteria include model formulation techniques and concept, 
simulator and model implementation, convergence performance, accuracy and validity region, and model parameter extraction, among others [1]. Recently, a comprehensive work was performed to review the criteria and the concerns on modeling problems in power semiconductor device [2]. The study raised issues about the uncertainty in models for circuit simulation, which stems from the need to simultaneously fulfill contradicting requirements like high accuracy, low demand of computing power, and easily accessible model parameters. Also, the study concluded that a favorable trade-off between these contradicting requirements is necessary.

Actually, a model is valuable for power circuit designers only if it fulfills the objective of the circuit designers, whether the objective is to achieve a high accuracy or a fast computation. In this instance, to the engineers who actually design power circuits, the degree of accuracy versus calculation time should be specified for circuit simulation. However, the criteria are interdependent in a complex way and overlapping, therefore, in reality, some subjective judgment is inevitable to find the level of the importance of the criteria. If this process of specification does not proceed in an organized and well-planned way, a more accidental decision may happen. Such an accidental procedure may lead to temporarily wrong developments and certainly slow down the overall progress. Therefore, a practical need for efficient power circuit designs is to devise an evaluation framework to find the best-fit model among various candidates for a specific purpose and objective.

In this paper we adopted uncertainty reasoning and the framework of decision-making under incomplete knowledge to manage the uncertainties. The framework produces a range of the model-fit index according to the circuit designers' subjective or $a$ priori specification on the importance of the performance criteria. In the next section, we discuss the framework and the method for deriving model-fit index of the models under different specification. Case studies illustrate the application of the framework using a priori specifications derived from [1] and [2].

\section{DECISION MAKING UNDER INCOMPLETE KNOWLEDGE}

One of the principal concerns of decision making is the development of analytical method for guiding the choice of a single course of action from among a series of alternatives. The classical decision theory provides a framework such that a decision-maker can select one of a number of strategies open to it. However, since circuit designers usually have different levels of knowledge and different specifications on the performance criteria to choose a model. Therefore, the appropriateness of the selected model will depend on the levels of importance of the criteria.

There are a few different frameworks of decision making, which are distinguished by the amount of information available about the "favorite level" with which the performance criterion is important to the circuit designers. In the problem of selecting and evaluating power semiconductor device models, circuit designer usually has some information about the performance criteria, but the information is not comprehensive enough to enable exact specification of the favorite level of the criteria. This situation is called decision making under incomplete knowledge [3].

\subsection{Decision under incomplete knowledge}

In decision making under incomplete knowledge, it is assumed that the performance criteria are ranked in terms of their importance such as $C_{1} \geq C_{2} \geq \ldots \geq C_{\mathrm{n}}$, where each $C_{\mathrm{i}}$ indicates one of the criteria. It is also assumed that, using subjective, a priori, specification of the models, the "favorite levels" of each model under each individual criterion are assigned[4]. Under these assumptions, the framework for decision making under incomplete knowledge, in an example of 4 models and 4 criteria, can be expressed in the following matrix form, "favorite matrix":

$$
\begin{aligned}
& \begin{array}{cccc}
C_{1} & C_{2} & C_{3} & C_{4}
\end{array} \\
& M_{1}\left(\begin{array}{llll}
A_{11} & A_{12} & A_{13} & A_{14}
\end{array}\right. \\
& \begin{array}{lllll}
M_{2} & A_{21} & A_{22} & A_{23} & A_{24}
\end{array} \\
& \begin{array}{lllll}
M_{3} & A_{31} & A_{32} & A_{33} & A_{34}
\end{array} \\
& M_{4}\left(\begin{array}{llll}
A_{41} & A_{42} & A_{43} & A_{44}
\end{array}\right)
\end{aligned}
$$

where, $\quad M_{\mathrm{i}}=\mathrm{i}^{\text {th }}$ model, $C_{\mathrm{j}}=\mathrm{j}^{\text {th }}$ performance criterion, and $A_{\mathrm{ij}}=$ subjective, a priori, favorite level of model $M_{\mathrm{i}}$ under criterion $C_{\mathrm{j}}$.

To apply this decision framework, circuit designers should (a) rank, roughly or precisely, the importance of the criteria for their specific circuit simulations and design environments, and (b) assign the "favorite levels" of the models under each criterion. 
As an example for assigning the "favorite levels" for a criterion of accuracy $(C=$ accuracy $)$, an examination of the modeling types such as behavioral, numerical, analytical, and hybrid type, could be a good starting point. If the designers can assign their favorite levels, in the range of $(1,5)$ for the accuracy, such as $A$ [behavioral model] $=4$, $A$ [analytical model $]=5, A[$ numerical model $]=3$, and so on, then, they use this favorite level to form a favorite matrix for the accuracy when the model type is identified.

In the application of the decision framework, there are two practical methods in deriving the range of expected performance, "model-fit index": weak and strict ranking of the importance of the criteria. The model-fit index indicates how fitting or appropriate a model is under a chosen environment or specified purpose.

Model-fit index given weak ranking of the importance of the criteria: This situation assumes that the circuit designer is able to roughly, perhaps without much assurance, rank the importance of the criteria for a circuit simulation purpose. In this situation, the expected model-fit index of a model $i$ under a criterion $j, F_{\mathrm{ij}}$, can be found by computing partial averages as shown below [3].

$$
F_{i j}=\frac{1}{j} \sum_{k=1}^{j} A_{i k}, j=1, \ldots, n, \text { and } i=1, \ldots, m \text {. }
$$

where $m$ indicates the number of candidate models and $n$, the number of criteria. For each candidate model, the largest such partial average is the maximum expected outcome of model fit-index and the smallest, the minimum.

Model-fit index given strict ranking of the importance of the criteria: This situation has more information about ranking of importance on the criteria, i.e.,

$$
C_{j}-C_{j+1} \geq K_{j}, j=1, \ldots, n
$$

where, $C_{n+1}=0$ and $K_{j}$ are positive constants.

The expected model-fit index for this situation can be calculated by the following equation suggested in [3]. For each candidate model, the largest of these gives the maximum model-fit index and the smallest the minimum.

$$
F_{i j}=\frac{1}{j} \sum_{l=1}^{j} A_{i l}\left(1-\sum_{l=1}^{j} K_{l}\right)+\sum_{l=1}^{j} K_{l} A_{i l}
$$

Fig.1 illustrates the framework of model selection. In the framework, a designer ranks the criteria on candidate models and assigns the favorite level to form the favorite matrix helped by a priori or subjective information. Then model fit index is calculated based on the way the criteria are ranked, weakly or strongly. Designer chooses a model that shows the highest model fit index. Detailed discussion on the framework follows.

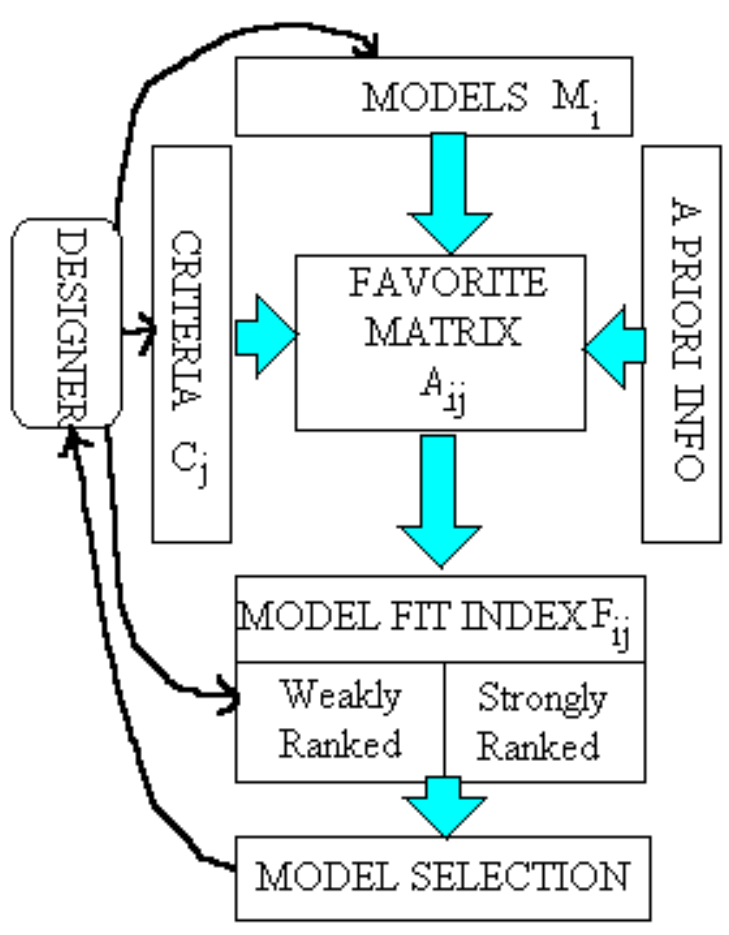

Fig.1. Illustration of Model Selection Framework

\subsection{Components for Favorite Matrix}

To apply the decision making under incomplete knowledge, it is necessary to provide necessary information on the models and a priori or subjective specifications. Specifically, in the favorite matrix construction, we need the following three components: model information, performance criteria, and a priori or subjective specification on the model's performance under the criteria.

Model Information: We assume that each model provides information on model type, number of parameters, and simulator types.

Performance Criteria: From the various references [5-11], in addition to [1] and [2], the most common criteria mentioned and applied are, in an arbitrary 
order, the following five: accuracy, computation time, number of parameter, parameter extraction, and simulator types supported. Since the purpose of using a model is not the same, the order of the importance of the criteria are not the same. For instance, accuracy is the most important criterion for some, but computation time is more important than other criteria for others. In other words, the rank of the importance of the criteria is different in different situations.

A priori or Subjective Specification: To form a favorite matrix, a priori knowledge base or circuit designer's subjective specification on the above two components should be provided. For a priori specification, we referred [1] that studied tens of power diode models and [2] that attempted to find "objective" expected performance, which inevitably included author's subjective favorites, of various types of power semiconductor models. We construct a priori specification tables by combining the findings of these two references.

\subsection{Formation of a priori specification table}

Following [2], we are able to find the favorite levels of models under the following three criteria: accuracy, computation time, and parameter extraction. The numerical values of the favorite level are classified into five groups: 5 for excellence, 4 for very good, 3 for good, 2 for fair, and 1 for poor. Table I shows a priori specification table for the three criteria in terms of model types.

Table I. A priori specification table for three criteria.

\begin{tabular}{|c|c|c|c|}
\hline $\begin{array}{l}\text { Model } \\
\text { Type }\end{array}$ & Accuracy & $\begin{array}{l}\text { Computation } \\
\text { Time }\end{array}$ & $\begin{array}{l}\text { Parameter } \\
\text { Extraction }\end{array}$ \\
\hline $\mathrm{M}_{1}$ & 5 & 2 & 1 \\
\hline $\mathrm{M}_{2}$ & 2 & 3 & 3 \\
\hline $\mathrm{M}_{3}$ & 2 & 3 & 3 \\
\hline $\mathrm{M}_{4}$ & 4 & 1 & 2 \\
\hline $\mathrm{M}_{5}$ & 1 & 5 & 5 \\
\hline
\end{tabular}

Note: $\mathrm{M}_{1}$ : Functional, $\mathrm{M}_{2}$ : Approximation Solution,

$\mathrm{M}_{3}$ : Transformation, $\mathrm{M}_{4}$ : Lumped Model, $\mathrm{M}_{5}$ : Numerical Solution.

Table II. A priori specification table for number of parameter and simulator type

\begin{tabular}{|l|l|l|l|}
\hline \multicolumn{2}{|l|}{ number of parameter } & \multicolumn{2}{l|}{ simulator type } \\
\hline$<10$ & 5 & All & 5 \\
$11-20$ & 4 & SPICE \& SABER & 4 \\
$21-30$ & 3 & SPICE only & 3 \\
$31-40$ & 2 & SABER only & 2 \\
$>40$ & 1 & Other & 1 \\
\hline
\end{tabular}

The a priori specification table for the other two criteria, the number of parameter and the simulator type, is similarly formed using [1] as shown in Table II. In this table, the classification and the favorite levels are arbitrary divided and chosen.

\section{APPLICATION EXAMPLES}

We applied the framework of decision making under incomplete knowledge to determine the model-fix indices, under two different scenarios, for the four models of power diode selected from [1]. Two of the models are analytical types and the other two, numerical types. Information of the models is tabulated in Table III. The five criteria and $a$ priori specification tables I and II are used in the case study.

Table III. Selected Power Diode Models

\begin{tabular}{|l|l|l|l|}
\hline No. & $\begin{array}{l}\text { Model } \\
\text { Type }\end{array}$ & Simulator & $\begin{array}{l}\text { Number of } \\
\text { Parameter }\end{array}$ \\
\hline $1^{\mathrm{a}}$ & Analytical & $\begin{array}{l}\text { PSPICE, } \\
\text { SABER }\end{array}$ & 6 \\
\hline $2^{\mathrm{b}}$ & Analytical & SABER & 59 \\
\hline $3^{\mathrm{c}}$ & Numerical & SABER & 26 \\
\hline $4^{\mathrm{d}}$ & Numerical & Other & 6 \\
\hline
\end{tabular}

Note: $a$ : model \#8 in [1], $b$ : model \#9 in [1], $c$ : model \# 12 in [1], $d$ : model \# 14 in [1].

\subsection{Case 1}

In case 1, we assumed the criteria are ranked in the order of accuracy $\left(C_{1}\right)$, computation time $\left(C_{2}\right)$, parameter extraction $\left(C_{3}\right)$, number of parameter $\left(C_{4}\right)$, and simulator type $\left(C_{5}\right)$. Using the model information given in Table III and the a priori specifications provided in Tables I and II, we formed the favorite matrix as shown in Table IV.

Table IV. Favorite matrix for case 1

$$
\begin{aligned}
& \begin{array}{lllll}
C_{1} & C_{2} & C_{3} & C_{4} & C_{5}
\end{array} \\
& M_{1}\left(\begin{array}{lllll}
5 & 2 & 1 & 5 & 4
\end{array}\right) \\
& \begin{array}{l|lllll}
M_{2} & 5 & 2 & 1 & 1 & 2
\end{array} \\
& \begin{array}{l|lllll}
M_{3} & 1 & 5 & 5 & 3 & 2
\end{array} \\
& M_{4}\left(\begin{array}{lllll}
1 & 5 & 5 & 5 & 1
\end{array}\right)
\end{aligned}
$$

Note: $C_{1}$ : accuracy, $C_{2}$ : computation time, $C_{3}$ : parameter extraction, $C_{4}$ : number of parameter, and $C 5$ : simulator type

Weakly Ranked Criteria: When the criteria are weakly ranked, we apply equation (1) to calculate the 
expected model-fit index for each model. Calculations for model \#1 are illustrated below.

$$
\begin{aligned}
& F_{11}=5, \\
& F_{12}=\frac{5+2}{2}=3.5, \\
& F_{13}=\frac{5+2+1}{3}=2.7, \\
& F_{14}=\frac{5+2+1+5}{4}=3.3, \\
& F_{15}=\frac{5+2+1+5+4}{5}=3.4
\end{aligned}
$$

Therefore, for model $\# 1$, the range of the model-fit index is $(5.0,2.7)$. Model-fit indices for other models are similarly derived and the ranges of the model-fit indices of the models are

$F_{1}=(5.0,2.7), \quad F_{2}=(5.0,2.2), \quad F_{3}=(3.7,1.0)$, and $F_{4}=(4.0,1.0)$.

By observing the model-fit index ranges, one may choose either model \#1 or \#2 over the other two.

Strictly Ranked Criteria: Let's consider when the criteria are ranked in numbers such that $C_{1}=5, C_{2}=4$, $C_{3}=3, C_{4}=1.5$, and $C_{5}=1$. Then, we first calculate the constants $K_{\mathrm{j}}=C_{\mathrm{j}}-C_{\mathrm{j}+1}$ and derive them as $K_{1}=1, K_{2}=$ $1, K_{3}=1.5, K_{4}=0.5$, and $K_{5}=1 .\left(C_{6}\right.$ is assumed to be 0.) We then use equation (2) for model-fit index calculation. An illustration for model \#1 is shown below.

$$
\begin{aligned}
F_{11}= & 5(1-1)+1 \cdot 5=5, \\
F_{12}= & \frac{7}{2}(1-2)+1 \cdot 5+1 \cdot 2=3.5, \\
F_{13}= & \frac{8}{3}(1-3.5)+1 \cdot 5+1 \cdot 2+(1.5) \cdot 1=1.8, \\
F_{14}= & \frac{13}{4}(1-4)+1 \cdot 5+1 \cdot 2+(1.5) \cdot 1+(0.5) \cdot 5 \\
= & 1.3 \\
F_{15}= & \frac{17}{5}(1-5)+1 \cdot 5+1 \cdot 2+(1.5) \cdot 1+(0.5) \cdot 5 \\
& +1 \cdot 4=1.4
\end{aligned}
$$

Therefore, the range of the model-fit index of model \#1 is $(5.0,1.3)$. The other model-fit indices are similarly derived, and they are:

$F_{1}=(5.0,1.3), \quad F_{2}=(5.0,1.8), \quad F_{3}=(4.5,1.0)$, and $F_{4}=(4.3,1.0)$
By observing the ranges, it is apparent that the choice is either model \#1 or \#2.

\subsection{Case 2}

In case 2, the criteria are ranked differently: the order now is computation time $\left(C_{1}\right)$, parameter extraction $\left(C_{2}\right)$, simulator types $\left(C_{3}\right)$, accuracy $\left(C_{4}\right)$, and number of parameter $\left(C_{5}\right)$. Using the same information on the selected models and the a priori specification tables, we construct another favorite matrix as shown in Table V.

Table V. Favorite matrix for case 2

$$
\begin{aligned}
& \begin{array}{lllll}
C_{1} & C_{2} & C_{3} & C_{4} & C_{5}
\end{array} \\
& \begin{array}{l}
M_{1} \\
M_{2} \\
M_{3} \\
M_{4}
\end{array}\left(\begin{array}{lllll}
2 & 1 & 4 & 5 & 5 \\
2 & 1 & 2 & 5 & 1 \\
5 & 5 & 2 & 1 & 3 \\
5 & 5 & 1 & 1 & 5
\end{array}\right)
\end{aligned}
$$

Note: $C_{1}$ : computation time, $C_{2}$ : parameter extraction, $C_{3}$ : simulator type, $C_{4}$ : accuracy, and C5: number of parameter

Weakly Ranked Criteria: When criteria are weakly ranked, we use equation (1) to calculate the ranges of the model-fit indices of the models, and they are:

$F_{1}=(3.4,1.5), \quad F_{2}=(2.5,1.7), \quad F_{3}=(5.0,3.2)$, and $F_{4}=(5.0,3.0)$.

Therefore, in this scenario, one surely chooses either model \#3 or \#4 over \#1 and \#2.

Strictly Ranked Criteria: For the strictly ranked criteria such as $\mathrm{C} 1=5, C_{2}=4, \mathrm{C} 3=3, C_{4}=1.5$, and C5 $=1$, the constants are derived as $K_{1}=1, K_{2}=1, K_{3}=1$, $K_{4}=1.5$, and $K_{5}=0.5$. By using equation (2), the ranges of the model-fit indices are calculated and they are:

$F_{1}=(4.0,1.5), \quad F_{2}=(4.2,1.5), \quad F_{3}=(5.0,2.1), \quad$ and $\mathrm{F} 3=(5.0,2.0)$.

Similarly, the apparent choice is either model \#3 or \#4.

\subsection{Observations}

As illustrated in the case study, the model-fit index of a model changes when the rank of the criteria is changed. Therefore, using the suggested framework, circuit designers can utilize their own rank of the criteria and the favorite levels, to select the best-fit model of their purposes and surrounding conditions. Also, we can observe that the outcome of the model- 
fit index is more influenced by the order of the criteria than the way they are ranked, either weakly or strictly. This frame of decision making under incomplete knowledge is hoped to serve as a practical tool for circuit designers who just want to find and select the best model for their various objectives and goals.

\section{CONCLUSIONS}

The uncertainty in modeling and simulation in power device comes from many different sources. This uncertainty brings a question of how to choose the best-fit model for a specific purpose and environment of a circuit designer. In this paper we adopted the framework of decision making under incomplete knowledge to accommodate the uncertainties around the power device model evaluation and selection. The framework produces a range of the model-fit index for the candidate models according to the circuit designer's subjective rank of the performance criteria. Case studies are performed for practical applications of the framework. It is hoped that circuit designers can select the best-fit model of their objectives by the numerical framework of decision making under incomplete knowledge.

\section{REFERENCES}

[1] C. M. Tan, K. J. Tseng, "Using Power Diode Models for Circuit Simulations - A Comprehensive Review," IEEE Transactions on Power Electronics, vol. 46, no. 3, pp. 637645,June 1999.

[2] Rainer Kraus, H. J. Mattausch, "STATUS and Trends of Power Semiconductor Device Models for Circuit Simulation," IEEE Transactions on Power Electronics, vol. 13, no. 3, pp. 452-464, May 1998.

[3] Z. W. Kmietowicz, A. D. Pearman, Decision Theory and Incomplete Knowledge, Hampshire, UK: Gower Publishing Company Limited, 1981

[4] C. J. Kim, B. D. Russell, K. L. Watson, "A Parameter-Based Process for Selecting High Impedance Fault Detection Techniques using Decision Making under Incomplete Knowledge," IEEE Transactions on Power Delivery, vol.5, no. 3, pp. 1314-1320, July 1990.

[5] I. K. Budihardjo, P. O. Lauritzen, H. A. Mantooth, " Performance Requirements for Power MOSFET Models," IEEE Transactions on Power Electronics, Vol. 12, No. 1, pp. 36-45, January 1997.

[6] K. Shenai, "A Circuit Simulation Model for HighFrequency Power MOSFETs," IEEE
Transactions on Power Electronics, Vol. 6, No. 3, pp. 539-547, 1991.

[7] I. K. Budihardjo, P. O. Lauritzen, "A LumpedCharge Power MOSFET Model including Parameter Extraction," IEEE Transactions on Power Electronics, Vol. 10, No. 3, pp. 379-387, May 1995.

[8] R. A. Wunderlich, P. K. Ghosh, "Modeling the Gate more Accurately for Power MOSFETs," IEEE Transactions on Power Electronics, Vol. 9, No. 1, pp. 105-111, January 1994.

[9] H. A. Mantooth, J. L. Duliere, "A Unified Diode Model for Circuit Simulator," IEEE Transactions on Power Electronics, Vol. 12, No. 5, pp. 816823, September 1997.

[10]Y. C. Liang, V. J. Gosbell, "Diode Forward and Reverse Recovery Model for Power Electronic SPICE Simulations," IEEE Transactions on Power Electronics, Vol. 5, No. 3, pp. 346-356, July 1990.

[11]P. O. Lauritzen, C. L. Ma, "A Simple Diode Model with Reverse Recovery," IEEE Transactions on Power Electronics, Vol. 6, No. 2, pp. 188-191, April 1991.

\section{BIOGRAPHIES}

C. J. Kim received a Ph.D. degree from Texas A\&M University in 1989. From 1990 to 1994 he was research faculty member for the Power System Automation Laboratory at Texas A\&M University. From 1994 to 1998, he was an assistant professor at the University of Suwon. Since 1999, he is with Department of Electrical Engineering at Howard University. Dr. Kim's research interests include power electronics simulation and intelligent systems application to incipient failure detection.

Peter Bofah received his Ph.D. degree in electrical engineering from Howard University, Washington, DC in 1985. He received $\mathrm{BS}(\mathrm{EE})$ and $\mathrm{MS}(\mathrm{EE})$ both from the University of Nebraska-Lincoln. He is presently a faculty member with the Department of Electrical Engineering at Howard University. Dr. Bofah's research interests include power electronics, large flexible structures modeling and control, power electronics and drives, and energy systems. His teaching areas include power electronics, energy systems, systems theory, electronics and design. 